\title{
Tratamiento del libro de texto en la historia del sistema educativo español como mediador didáctico ent la educación musical
}

\author{
Sonsoles Ramos Ahijado (Universidad de Salamanca, Espanha) \\ sonsolesra@usal.es \\ Ana María Botella Nicolás (Universidad de Valencia, Espanha) \\ ana.maria.botella@uv.es \\ José Luis Rodríguez Pérez (Centro de Magisterio Virgen de Europa, Espanha) \\ josel.rodriguez@magisteriolalinea.com
}

\begin{abstract}
Resumen: En este trabajo se analizan las conexiones entre la educación y los libros de texto escolares, identificando ambos conceptos y sus características, con especial interés en la asignatura de música. El objetivo fue comprender el tratamiento de dichos mediadores didácticos a lo largo de la historia del sistema educativo español, constatando la importancia otorgada y su concreción en la legislación educativa. La metodología utilizada fue cualitativa a través de una revisión documental. El corpus de la investigación fue el análisis de los preceptos legislativos educativos, valorando tanto la educación musical como los libros de texto, por medio de la clasificación. Se concluye presentando la dimensión del libro de texto de música como mediador didáctico, considerando las principales herramientas de análisis, para así facilitar con el proceso de su elección, establecer un marco de referencia y contribuir a la mejora educativa.
\end{abstract}

Palabras clave: Educación musical; libro de texto; historia de la educación.

\section{Treatment of the textbook in the history of the Spanish educational system and as a didactic mediator in music education.}

\begin{abstract}
This paper analyzes the connections between education and school textbooks, identifying both concepts and their characteristics, with special interest in the subject of music. The objective was to understand the treatment of such didactic mediators throughout the history of the Spanish educational system, noting the importance given and their concretion in the educational legislation. The methodology used was qualitative through a documentary review. The research's corpus was the analysis of educational legislative, valuing music education and textbooks, through the classification. It concludes by presenting the dimension of music textbook as a teaching mediator, considering the main analysis tools, to facilitate the choice process, in order to establish a framework and contribute to educational improvement.
\end{abstract}

Keywords: Music education; textbooks; history of education.

\section{Tratamento do livro de texto na história do sistema educacional espanhol como mediador didático na educação musical}

Resumo: Neste artigo, analisam-se as conexões entre a educação e os livros escolares, identificando os conceitos e suas características, com especial interesse no tema da música. O objetivo foi compreender o tratamento dos ditos mediadores didáticos ao longo da história do sistema educacional espanhol, confirmando a importância dada e sua concretização na legislação educacional. A metodologia utilizada foi qualitativa através de uma revisão documental. O corpus da pesquisa foi a análise de preceitos legislativos educacionais, valorizando tanto a formação musical quanto os livros didáticos, por meio da classificação. Conclui apresentando a dimensão do livro didático de música como mediador didático, considerando as principais ferramentas de análise, a fim de facilitar com o processo de sua escolha, estabelecer um quadro de referência e contribuir para a melhoria educacional.

Palavras-chave: Educação musical; livro de texto; história da educação.

\section{Introducción}

La educación, entendida como proceso dinámico de asimilación y adaptación, ha estado presente a lo largo de toda la historia del ser humano. No obstante, debemos esperar hasta la génesis de los sistemas escolares nacionales, para hallar el uso de su vocablo en la 
lengua castellana. Acaece con la finalidad de favorecer la gestión de propósitos individuales y sociales (BERNABEU, 1998).

La educación es un proceso bidireccional en el que participan los docentes y educandos, en directa interacción, e implicando a toda la comunidad educativa, en donde se favorece el desarrollo de conocimientos, costumbres, procedimientos, capacidades, habilidades, actitudes, valores y normas, atendiendo en la actualidad, al saber, saber hacer, y saber ser y estar (SARRAMONA, 1998). Por tanto, implica una adaptación social permanente, "con un carácter complejo, multifactorial y variable” (CABRERA, 2009, p. 72).

En los primeros años de vida del ser humano se van forjando los cimientos sociales, afectivos, cognitivos, morales, culturales y emocionales, que modelarán a lo largo de toda su vida, por lo que la educación obligatoria ${ }^{1}$, será un estadio fundamental donde asentar las bases de su ulterior desarrollo (SANUY; GONZÁLEZ, 1969).

La educación se considera necesaria para la sociedad, considerando aspectos biológicos, psicológicos y sociológicos, puesto que aflora por la necesidad de supervivencia del ser humano ante la precariedad primitiva, en la búsqueda de conocimientos, técnicas e instrumentos de superación, incidiendo en su carácter de formación permanente. Es un fenómeno universal, ya que supera las barreras temporales y espaciales, al generarse a lo largo de la historia en las diferentes civilizaciones y pueblos distribuidos por todas las latitudes del planeta, contribuyendo a la mejora las relaciones interpersonales, y complejo, al estar condicionado por numerosos factores (SARRAMONA, 1989).

Se genera de forma integral, superando las expresiones verbales, por lo que también aglutina todas nuestras acciones, sentimientos y actitudes, consideradas en el ámbito educativo como currículo oculto ${ }^{2}$. Por consiguiente, se debe tener presente en todo proceso formativo del ser humano, la trascendencia de contemplar los sentimientos, las inquietudes estéticas y la sensibilidad (FREGA, 1996).

Según Domingo (2009, p. 9), "la educación básica se considera un derecho social que el estado tiene la obligación de asegurar a todo el mundo, a la vez que pasa a ser un factor básico para la cohesión social y para el progreso de las personas y de los países”. De este modo, es imprescindible favorecer el proceso de enseñanza y aprendizaje, donde "el alumno/a puede implicarse en un proceso abierto de intercambio y negociación de significados siempre que los nuevos contenidos provoquen la activación de sus esquemas habituales de pensar y actuar" (PÉREZ, 1993a, p. 74). Por ende, se valora la significación de la instrucción tanto primaria (de 6 a 11 años) como secundaria (de 12 a 16) para el desarrollo integral de las personas, puesto que "la universalización de la educación primaria, en primer lugar, y la generalización de la educación secundaria en segundo lugar suponen un salto cualitativo y, sobre todo, cuantitativo importantísimo” (DOMINGO, 2009, p. 9).

\section{Dimensiones y modalidades educativas}

La educación muestra cuatro dimensiones: moral, social, económica y política (BERNABEU, 1998).La dimensión moral se asienta en las teorías que se pronuncian a través de los conceptos de autonomía y heteronomía. La primera se sustenta en la libertad y dignidad humana, mientras que la segunda contempla la adquisición de unos hábitos admitidos socialmente. La autonomía moral se considera como una evolución de las normas morales, por lo que "para despertar la conciencia moral de los educandos, resultan decisivos la actitud dialogante del profesor y el funcionamiento democrático de la comunidad escolar" (BERNABEU, 1998, p. 29), desechando tanto la ignorancia como la manipulación, y estimando una clarificación de valores. 
La dimensión social persigue adiestrar al ser humano para la sociedad, actuando en situaciones de conflicto o degradación de la vida del hombre, partiendo de las ideas de autores como Pestalozzi, Natorp o Nohl (BERNABEU, 1998). Por ello, "desde que la configuración social de la especie se convierte en un factor decisivo de la hominización y en especial de la humanización del hombre, la educación, en un sentido amplio, cumple una ineludible función de socialización” (PÉREZ, 1993b, p. 17).

Actualmente, esta dimensión presenta gran importancia debido al Estado del bienestar, puesto que se pretende paliar las circunstancias de marginación y necesidad, por lo que en la educación formal responde a contenidos como la educación ambiental, del consumidor o de la salud, entre otros, y destaca la figura del voluntariado.

Sarramona y Rodríguez (2010, p. 4) expresan que "la educación escolar incide directamente sobre los valores sociales, sobre el conjunto de la cultura y sobre la personalidad individual de cada ciudadano, por ello mismo ha de ser una responsabilidad colectiva y compartida".

La dimensión económica parte de la teoría del capital humano, enunciada en la década de los años sesenta del siglo XX en los Estados Unidos de América, bajo la premisa de que el hombre educado es más productivo cuantitativa y cualitativamente, puesto que para la totalidad de los sociólogos, "el objetivo básico y prioritario de la socialización de los alumnos/as en la escuela es prepararlos para su incorporación futura en el mundo del trabajo" (PÉREZ, 1993b, p. 19).

La dimensión política prioriza en conexionar la educación con la vida pública, y más concretamente con el Estado, puesto que se integra en el proceso educativo el conocimiento de lo público, bajo una finalidad de mejora. Con el desarrollo de los sistemas educativos se institucionaliza el proceso de enseñanza y aprendizaje, donde es planificado y estructurado por el Estado, impulsando el patrimonio social, cultural e histórico de los pueblos.

Cabrera (2009), muestra la terminología de realidad educativa, desde una concepción más amplia, fusionando los agentes, factores y procesos que forman la educación con las teorías y valores inmersos en el proceso educativo.

Por otra parte, existen tres modalidades educativas: educación informal, no formal $\mathrm{y}$ formal.

La educación informal acaece externamente al ámbito educativo, prescindiéndose de todo tipo de planificación. Se considera como una de las mejores alternativas para el desarrollo de habilidades donde se priorice la práctica sobre la teoría, puesto que al prescindir del currículo, "permite mayor libertad a la hora de definir objetivos, adaptables a las circunstancias” (IBÁÑNEZ; VICENT;ASENSIO, 2012, p. 4).

La educación no formal precisa de planificaciones formalizadas, pero dichos programas se inscriben en ámbitos no escolares, y por ende, no contemplados en la legislación educativa. Al converger el ámbito educativo con la música, hallamos las Escuelas de Música y las Academias, donde "el núcleo central de sus enseñanzas es el estudio de un instrumento y en estos se incluye el instrumento más perfecto y delicado que es la voz humana" (ORIOL, 2004, p. 2).

La educación formal se contempla al generarse en centros educativos, desarrollando un currículo establecido.

Si vinculamos la educación formal con la educación musical, encontramos su aplicación principalmente, en los Centros de Educación Primaria y Secundaria, en los Conservatorios de Música y en la Universidad, aunque presentando finalidades educativas específicas. En los Conservatorios de Música se aborda el proceso de enseñanza y aprendizaje de la música por el arte, mientras que en los Centros de Educación Primaria se trabaja por 
medio del arte, buscándose en este último caso, el desarrollo integral del ser humano, por lo que se persiguen progresos más intangibles (MARTENOT, 1993). Efectivamente, puesto que debemos entender la educación musical "no solamente como la capacitación para percibir el mensaje musical, sino como un importante elemento de formación integral de la personalidad” (BARNIOL, 1998, p. 85).

De este modo, la educación confluye con el libro de texto, ya que es el material curricular usado tradicionalmente en mayor cuantía utilizado por excelencia en el proceso de enseñanza y aprendizaje, aunque en la actualidad, encontramos docentes que declinan su uso a favor de otros mediadores didácticos. Identificamos por materiales curriculares, "aquellos objetos, libros o instrumentos que se utilizan en las aulas, que los docentes emplean para desarrollar su enseñanza y estimular el aprendizaje de los estudiantes” (BLANCO, 1994, p. 263).

El libro de texto es un recurso didáctico utilizado tradicionalmente en el proceso de enseñanza y aprendizaje en todos los países, arraigando su aplicación desde prácticamente la universalización de la educación. Se consideran como "los mediadores curriculares básicos que se utilizan en nuestras escuelas occidentales” (CABERO et al., 1995, p. 21).

El uso del libro de texto escolar muestra una gran aprobación, constatándose por las elevadas cifras tanto de facturación como de número de ejemplares vendidos, ya que según "la Asociación Nacional de Editores de Libros y Material de Enseñanza (ANELE) ${ }^{3}$, la edición de libros de texto no universitario supone una quinta parte de la facturación total del sector del libro en España” (GARCÍA; ARREDONDO, 2006, p. 6). Para considerar su magnitud, debemos tener presente que las principales empresas editoras de libros de texto pertenecen a influyentes grupos empresariales que también se dedican al control y a la venta de la información, así como a las redes de comunicación (MARTÍNEZ, 2008).

Este mediador didáctico presenta gran trascendencia para los docentes, principalmente en los más conservadores, por ello, cuando es empleado en exclusividad, y no con una función de apoyo, puede llegar a regular el trabajo docente, mermando su autonomía y deteriorando su rol profesional (CARBONELL, 2001).

Por su parte, Adell y Bernabé (2006) dividen el ciclo de los libros de texto escolares en cuatro momentos, recogiendo a los implicados. El primero corresponde al diseño del currículo por la administración central y autonómica. El segundo es la creación, donde las editoriales encargan a los autores su desarrollo atendiendo a lo estipulado en la legislación educativa. El tercero se enmarca en la distribución y venta. El cuarto se centra en la propuesta didáctica del libro de texto escolar, establecida por el docente.

Martínez (2008) presenta la aplicación del libro de texto escolar como una praxis social donde se genera una interacción particular de un modelo político, económico, cultural y de la organización de políticas públicas en el Estado. No obstante, este material pedagógico es considerado con cierta obsolescencia por parte de la sociedad, debido a la facilidad con que se obtiene información en la sociedad contemporánea, caracterizada por la producción y consumo de información (ADELL; BERNABÉ, 2006).

Considerando dicho argumento, Carbonell (2001) señala que la aplicación del libro escolar genera principalmente dos posturas. Por una parte, aquellos docentes que mantienen un discurso moderado, aceptando el libro de texto como cualquier otro recurso material, pero negando su condición de exclusividad. Por otra, los maestros con un ideal más progresista, mostrándose a favor de su abolición.

En la actualidad, los libros de texto se elaboran por un grupo de docentes amparados bajo una editorial (IVANOVA, 2012), estableciendo el modo de mediación entre docentes y discentes, incidiendo en la labor del profesorado. Además, debemos tener presente que 
la auténtica autonomía de los docentes sólo puede estar limitada por la responsabilidad (LÓPEZ, 2007). Por ende, consideramos de vital importancia atender a algún modelo de análisis en el proceso de la elección del libro de texto escolar, puesto que el material seleccionado va a definir parte del proceso de enseñanza y aprendizaje.

\section{El libro de texto en la historia del sistema educativo español}

El primer precepto legislativo que ilustra el uso de los libros de texto en la historia del sistema educativo español, aludiendo a la instrucción primaria, lo hallamos en el Reglamento Provisional de las Escuelas Públicas de Instrucción Primaria Elemental de 1838. Se contempla al autorizar tanto a los docentes como a las Comisiones locales, la selección de los libros escolares, previa aprobación por parte de la Comisión provincial, siendo esta última la encargada de informar al Gobierno. Además, se matizan las razones por las que pudieran incurrir en una errónea elección de los mismos y se valora tanto la sustitución como la adaptación de dichos mediadores didácticos para las clases sociales más desfavorecidas (IMPRENTA NACIONAL, 1838, p. 13-31)"

Después de haber reflexionado detenidamente sobre las ventajas y desventajas
de señalar o no libros de texto, ha parecido conveniente autorizar a los Maestros y
Comisiones locales para que elijan los que les parezcan mejores, con el conocimiento
siempre é implícita aprobación de la respectiva Comisión provincial, que a su vez dará
noticia al Gobierno de los libros de uso en las Escuelas. Esta disposición, arriesgada á
primera vista, deja de serlo en el supuesto de que los Maestros y Comisiones cumplan
con su deber. Si no cumplen, nada se adelantaría con ordenar otra cosa que pudiera
igualmente dejarse de cumplir. El riesgo de que en las Escuelas se haga uso de malos
libros, ha de provenir necesariamente de una de tres causas; ó de malos principios
religiosos, morales o políticos, ó de ignorancia, ó de falta de medios para adquirir
libros buenos. [...] Atendida la falta general de libros uniformes en las clases pobres, convendrá que los Ayuntamientos y Comisiones proporcionen a los Maestros series de lecciones impresas en hojas sueltas, que puedan pegarse sobre cartones ó tablas, y sirvan para que lean todos los niños de una sección colocados delante de ellas.

Carbonell (2001, p. 76), considerando la instrucción secundaria y superior, ubica la adopción de los libros de texto en el sistema educativo español, a partir de la promulgación del Real Decreto de 17 de septiembre de 1845, por el que se aprueba el Plan General de Estudios para la instrucción pública del reino, conocido como Plan Pidal5, con objeto de "sistematizar los avances de la ciencia moderna, proporcionar los conocimientos y orientaciones básicas para llegar a dominar una materia y convertirse en el punto de referencia para la enseñanza del profesorado y el estudio del alumnado”.

En el documento Real Decreto de 17 de septiembre de 1845 se encuentra una exigua mención sobre los libros de texto en el artículo 134 del Título primero de la Sección cuarta (GM 4029, 1845, p. 5), donde se muestran las funciones del Consejo de instrucción pública:

Art. 134. El Consejo de instrucción pública dará su dictamen cuando sea consultado por el Gobierno:

$1^{0}$. Sobre creación, conservación y supresión de establecimientos de instrucción pública.

$2^{\circ}$. Sobre los métodos de enseñanza y libros de texto.

$3^{\circ}$. Sobre los reglamentos de toda clase de escuelas.

$4^{\mathrm{o}}$. Sobre la provisión de cátedras. 
$5^{\mathrm{o}}$. Sobre la antigüedad y clasificación de los profesores.

$6^{\circ}$. Sobre remoción de los catedráticos propietarios.

$7^{\circ}$. Sobre las cuestiones que se susciten relativas al gobierno interior de los establecimientos y penas académicas.

$8^{\circ}$. Sobre los demás puntos relativos a la enseñanza en que el Gobierno tenga por conveniente oírle.

En la Ley de 9 de septiembre de 1857 de Instrucción Pública, Ley Moyano, se aprecia reseña en el Título quinto que versa íntegramente sobre estos mediadores didácticos, desarrollando su tipología y regulación, otorgando gran importancia tanto a la formación Moral y Religiosa como de la Gramática y Ortografía, y matizando la obligatoriedad de su aplicación en todos los niveles educativos, como expresa desde el artículo 86 al 93 (GM 1710, 1857, p. 2):

Art. 86. Todas las asignaturas de la primera y segunda enseñanza, las de las carreras profesionales y superiores y las de las facultades hasta el grado de Licenciado, se estudiarán por libros de texto; estos libros serán señalados en listas que el Gobierno publicará cada tres años.

En la Ley de 2 de junio de 1868 de Instrucción Primaria existen referencias, al emplear en exclusiva todo el capítulo segundo del Título primero a explicar la tipología, orientación, ideario y censura aplicada en la selección de los libros de texto utilizados en las Escuelas públicas y privadas. No acaece mención alguna a los mediadores didácticos de contenido musical, aunque previamente, en el artículo 14 del capítulo primero del citado Título, sí se alude a la práctica del canto, si bien de manera muy marginal (GM 156, 1868, p. 2):

Art. 24. Cada cinco años publicará el Gobierno la lista de los libros que deberán servir de texto en las Escuelas públicas y privadas de primera enseñanza.

(...) Art. 28. Se encomendará a las Reales Academias, según su respectivo instituto, la formación de ligeros epítomes de las materias que comprende la instrucción primaria, así para asegurar el acierto y la posible unidad en esta clase de obras, como para que se facilite su adquisición a todas las localidades, con grande economía de las familias y de los pueblos.

La alusión abajo, presente en los artículos séptimo y octavo del Real Decreto de 26 de octubre de 1901 dictamina la obligatoriedad de los libros para abordar los contenidos de Doctrina Cristiana, Gramática y Lectura, así como su regulación, en sendos grados de la instrucción primaria (GM 303, 1901, p. 498):

Art. 7. ${ }^{\circ}$ Tanto en el grado elemental como en el superior, constituye obligación ineludible señalar libros de texto para la enseñanza de la Doctrina Cristiana, de la Gramática y de la Lectura.

Art. 8. ${ }^{\circ}$ La Doctrina Cristiana se estudiará por el Catecismo que señalen los Prelados en sus respectivas diócesis; la Gramática, por el texto de la Real Academia Española de la Lengua, y la Lectura se ejercitará en libros que hayan sido aprobados por el Gobierno, previo informe del Consejo de Instrucción pública.

Curiosa mención en el artículo segundo de la Ley de 27 de agosto de 1932, intenta transformar el Consejo de Instrucción pública en el Consejo Nacional de Cultura, donde se dictamina la potestad del Consejo Nacional de Cultura para regular tanto la selección como la aprobación de los libros de texto que deban usarse en los Centros educativos, así como a 
establecer las pautas para la distribución de todos los materiales escolares (GM 254, 1932, p. 1818-1819):

Art. 2. ${ }^{\circ}$ El Consejo Nacional de Cultura, como organismo asesor del Ministerio, dictaminará necesariamente:

(...) f) Para fijar normas acerca de la distribución del material escolar.

g) Sobre la selección y aprobación de las obras que hayan que hayan de utilizarse como textos, así de lectura como de estudio, en los Centros de enseñanza.

Previamente a la instauración de la Dictadura franquista ${ }^{6}$, en plena contienda española, se encuentra otra referencia legislativa acerca del uso de los libros de texto en el sistema educativo español. Se manifiesta su normalización en la base quinta de la Ley de 20 de septiembre de $1938^{7}$ sobre Reforma de la Segunda Enseñanza, expresando la necesidad de su aprobación y regularización económica por parte de una Comisión especial establecida por el Ministerio de Educación Nacional.

Fue dictada en la sede del Ministerio de Educación en Vitoria, tras el nombramiento del primer gobierno de Francisco Franco el 1 de febrero de 1938, y rubricada por el caudillo en la ciudad de Burgos. Además, consideramos su relevancia por su marco transitorio hacia el asentamiento de la Dictadura militar, en el que se aprecia claramente el germen de su ideario y el tratamiento de la asignatura de música, bajo la denominación de Música y canto. Ésta se integra en el grupo de Educación Artística, Física y Patriótica, entendido como un complemento a las siete disciplinas fundamentales ${ }^{8}$, por lo que se reconoce explícitamente la naturaleza de dicha formación musical, destinada a la práctica de cantos con la finalidad de realzar el espíritu patriótico (BOE 85, 1938, p. 1390):

BASE V.- Libros de texto

Los libros docentes no podrán ser utilizados como textos por los Establecimientos de enseñanza, tanto del Estado como privados, sin que previamente hayan obtenido dictamen favorable a la Comisión especial designada por el Ministerio de Educación Nacional, constituida para tal objeto, quien asimismo fijará el precio máximo al que deberá ser vendido el libro para el público.

En la Ley de 17 de julio de 1945 sobre Educación Primaria ${ }^{9}$, observamos reseñas en el artículo 48, dentro del Capítulo VI, dedicado a los instrumentos pedagógicos. Se dictamina su regulación por el Ministerio de Educación Nacional, y manifiesta la necesidad de aprobar aquellos libros de texto de naturaleza religiosa al estamento eclesiástico. Como dato relevante, apreciamos el interés mostrado por parte del Ministerio de Educación Nacional para la mejora de los libros de texto, incentivando mediante concursos y premios de carácter anual a las mejores ediciones (BOE 199, 1945, p. 397):

Artículo cuarenta y ocho.- Los libros de uso escolar en todas las Escuelas españolas habrán de ser aprobados por el Ministerio de Educación Nacional, previos los asesoramientos técnicos en cuanto a su contenido y confección, sin lo cual no podrán utilizarse en la primera enseñanza, ni como textos ni como libros de lectura. En lo que afecten a doctrina religiosa, habrán de ser aprobados previamente por la Jerarquía eclesiástica, a la cual pertenece, además, el derecho de aprobar los libros de uso escolar en sus propias Escuelas. Los que tiendan a la formación del espíritu nacional habrán de ser aprobados por los organismos competentes.

Por tanto, para conseguir la aprobación por el Ministerio de Educación Nacional de un libro de texto, se precisa superar las siguientes condiciones (BOE 199, 1945, p. 397): 
a) Que se ajuste en su contenido a las normas de los cuestionarios oficiales;

b) Que su doctrina y espíritu estén en armonía con los artículos aplicables del Título I y del capítulo IV del Título II de la presente Ley;

c) Que sus cualidades materiales respondan a las exigencias pedagógicas en cuanto a papel, tipografía, tamaño, extensión e ilustraciones; y

d) Que su precio se acomode a la regulación que determine el Reglamento.

El Ministerio de Educación Nacional estimulará, mediante concursos y premios anuales, la edición de libros escolares.

La siguiente mención la hallamos en la Ley 14/1970, de 4 de agosto, General de Educación y Financiamiento de la Reforma Educativa ${ }^{10}$ (BOE 187, 1970, p. 12546), en la sección de disposiciones adicionales. Se expresa la función de supervisión del Ministerio de Educación y Ciencia de aquel material didáctico a utilizar en los niveles de Educación Preescolar, Educación General Básica, Formación Profesional de primero y segundo grados, y Bachillerato:

Quinta.- Los libros y material necesario para el desarrollo del sistema educativo en los niveles de Educación Preescolar, Educación General Básica, Formación Profesional de primero y segundo grados y Bachillerato, estarán sujetos a la supervisión del Ministerio de Educación y Ciencia, de acuerdo con las normas que reglamentariamente se establezcan.

En el Real Decreto 69/1981 ${ }^{11}$, de 9 de enero, de ordenación de la Educación General Básica y fijación de las enseñanzas mínimas para el Ciclo Inicial, aparece una referencia en el artículo noveno, por el que se dictamina que deben ajustarse a los contenidos mínimos fijados, "Artículo noveno.- Los libros y material didáctico del Ciclo Inicial deberán atenerse a las enseñanzas mínimas establecidas en el presenta Real Decreto” (BOE 15, 1981,p. 1097).

Otra alusión a los libros de texto la presenciamos en el artículo séptimo de la Orden de 17 de enero de 1981, por la que se regulan las enseñanzas de Educación Preescolar y del Ciclo Inicial de la Educación General Básica (BOE 18, 1981, p. 1385). Se matiza la obligatoriedad de ajustarse a los contenidos mínimos marcados en su anexo I, pero no acontece referencia explícita a los de contenido musical. Sin embargo, dictamina el tratamiento de la Lengua Castellana, Matemáticas y Experiencia Social y Natural, mediante materiales y libros de texto compartidos, por lo que consideramos su trascendencia, ya que se genera una aproximación al trabajo cooperativo:

Art. 7. ${ }^{\circ}$ 1. Los libros y material didáctico de Preescolar y Ciclo Inicial deberán ajustarse a los Niveles Básicos de Referencia que figuran en el Anexo I.

2. El desarrollo didáctico de las enseñanzas de Lengua Castellana, Matemáticas y Experiencia Social y Natural se hará en cuadernos de trabajo, libros de lectura y material de uso colectivo.

En el Real Decreto 710/1982 ${ }^{12}$, de 12 de febrero, por el que se fijan las enseñanzas mínimas para el ciclo medio de la Educación General Básica, aparece una reseña de los libros escolares en el artículo noveno, por el que se establece la obligatoriedad de que se ajusten a los contenidos mínimos fijados, "Artículo noveno.- Los libros y material didáctico del Ciclo Medio deberán atenerse a las enseñanzas mínimas establecidas en el presenta Real Decreto" (BOE 90, 1982, p. 9586). 
La siguiente mención a los mediadores didácticos aparece en la Orden de 6 de mayo de $1982^{13}$ por la que se regulan las enseñanzas del Ciclo Medio de la Educación General Básica. En su artículo séptimo, se dictamina la necesidad de ceñir el contenido de todos los materiales didácticos, incluidos los libros escolares, a la mencionada Orden, pero no acaece referencia explícita a los de música. Sin embargo, dispone el tratamiento de la Lengua Castellana, Matemáticas, Ciencias de la Naturaleza y Ciencias Sociales, mediante materiales y libros de texto compartidos. Por ello, consideramos su importancia, al igual que en la anterior Orden, puesto que acontece una aproximación al trabajo cooperativo (BOE 115, 1982, p. 12574):

Art. 7. ${ }^{\circ}$ Uno. Los libros y material didáctico del Ciclo Medio deberán ajustarse a los niveles básicos de referencia establecidos en la presente Orden ministerial.

Dos. El desarrollo didáctico de Lengua Castellana, Matemáticas, Ciencias de la Naturaleza y Ciencias Sociales podrá hacerse en Libros del Alumno, sin que su número sea superior a tres por área y Ciclo y en material didáctico de uso colectivo.

El Real Decreto 3087/1982 ${ }^{14}$, de 12 de noviembre, por el que se fijan las enseñanzas mínimas para el ciclo superior de Educación General Básica, dictamina en su artículo 10 que los libros de texto empleados en el Ciclo Superior de Educación General Básica deben adecuarse a los contenidos mínimos desarrollados en su anexo I. No obstante, la disposición transitoria establece la posibilidad de continuar utilizando los antiguos libros escolares durante los tres años académicos posteriores (BOE 280, 1982, p. 32011):

Artículo décimo.- Los libros y material didáctico del ciclo superior deberán atenerse a las enseñanzas mínimas establecidas en el presente Real Decreto.

\section{DISPOSICIÓN TRANSITORIA}

Los libros y material didáctico actualmente autorizado para los cursos sexto, séptimo y octavo de Educación General Básica podrán utilizarse, respectivamente, durante el curso mil novecientos ochenta y tres-ochenta y cuatro, ochenta y cuatro-ochenta y cinco y ochenta y cinco-ochenta y seis.

La última referencia a los libros de texto previa a la promulgación de la Ley Orgánica 1/1990 ${ }^{15}$, de 3 de octubre, de Ordenación General del Sistema Educativo, donde se establece la inclusión de la Educación Musical oficialmente en el currículo de la etapa de Educación Primaria, es presente en la Orden de 25 de noviembre de 1982, por la que se regulan las enseñanzas del Ciclo Superior de la Educación General Básica (BOE 291, 1982), que desarrolla el Real Decreto 3087/1982, de 12 de noviembre, por el que se fijan las enseñanzas mínimas para el ciclo superior de Educación General Básica (BOE 280, 1982).

Aparece una alusión a los libros de texto, expuesta en el artículo séptimo y en la disposición transitoria. Se dictamina la necesidad de adaptar progresivamente los materiales didácticos, y por ende, también los libros de texto, al contenido de los niveles de referencia, pero sin aludir de modo explícito a los de música. No obstante, se deja la posibilidad de usar los antiguos libros escolares durante el primer año en que entren en vigor los programas renovados. Por otra parte, limita el número de libros de texto a un máximo de tres por área y Ciclo, y considerando el uso compartido. Además, al igual que en las dos Órdenes anteriores, apreciamos un acercamiento al trabajo cooperativo en la Orden de 25 de noviembre de 1982 (BOE 291, 1982, p. 33446): 
Art. 7. ${ }^{\circ}$ Uno. Los libros y material didáctico de los cursos sexto, séptimo y octavo del Ciclo Superior de Educación General Básica deberán ajustarse progresivamente, y a medida que entren en vigor, a los niveles básicos de referencia establecidos en la presente Orden ministerial.

Dos, El desarrollo didáctico de los niveles básicos de referencia de cada una de las materias podrá hacerse en libros del alumno, sin que su número sea superior a tres por área y Ciclo, y en material de uso colectivo.

Teniendo en cuenta las características de la tecnología, el material didáctico que la desarrolle podrá integrarse en el de Ciencias de la Naturaleza o bien presentarlo con carácter independiente.

\section{DISPOSICIÓN TRANSITORIA}

Los libros y material didáctico autorizados para los cursos sexto, séptimo y octavo de Educación General Básica de acuerdo con las orientaciones pedagógicas de 6 de agosto de 1971, de 24 de octubre de 1977, de 6 de octubre de 1978 y 18 de febrero de 1980, podrán utilizarse durante el primer año académico en que se apliquen los programas renovados de cada uno de los cursos respectivamente.

\section{El libro de texto como mediador didáctico}

El libro de texto se muestra como uno de los recursos didácticos más longevos de la historia escolar (BRAGA; BELVER, 2016), siendo "un intermediario de incalculable valor entre las prescripciones derivadas de las decisiones políticas y los docentes, por una lado; y, por otro, es el intermediario entre los docentes y los estudiantes” (BLANCO, 1994, p. 265).

Además, como "suele ir acompañado de materiales complementarios que refuerzan las actividades (Compact Disc (CD), Digital Video Disc (DVD), programas informáticos...)” (VICENTE, 2010, p. 31), se considera la utilidad del libro de texto escolar como una herramienta adicional, sirviendo de apoyo en el proceso de enseñanza y aprendizaje. Efectivamente, puesto que "la gestión del libro de texto por parte del profesor puede, sin duda, permitir trabajar óptimamente con materiales con limitaciones” (ATIENZA, 2007, p. 570).

Considerando la utilidad de los libros de texto escolares de música, se ha abordado una revisión por diferentes autores como Martínez (1992), Blanco (1994), Cabero et al. (1995), Gutiérrez y Cansino (2001) e Ivanova (2012), proponiendo la aplicación de instrumentos donde se establecen una serie de criterios para facilitar el proceso de su elección.

Por ejemplo, se destaca, la pertinencia del lenguaje utilizado, puesto que se favorece la correcta comprensión por parte de los niños, infiriendo en la realización de las actividades con mayor precisión. También es fundamental observar si su metodología estimula el desarrollo de la creatividad, de la expresión y de la sensibilidad de los discentes, o por el contrario, se plantean unas actividades muy rígidas y dirigidas, limitando sus posibilidades. Además, es importante la relevancia de su validez científica y su tratamiento didáctico, puesto que se debe generar una progresión lineal en la gradación de la dificultad de las actividades, de acuerdo a las aptitudes de los educandos y atendiendo a todas las dimensiones de la educación musical.

Éste es un aspecto de vital importancia, puesto que en la praxis educativa, los libros de texto suponen para algunos profesores la referencia a partir de la cual gestionan su programación atendiendo a la interpretación de la editorial escogida. Efectivamente, puesto que "para evaluar un libro de texto se debe considerar el efecto que tendrá en el alumno o grupo de alumnos que lo pueden usar" (SCHIEFELBEIN; FARRÉS, 1991, p. 63), constatando la importancia de un riguroso análisis. 
Gutiérrez y Cansino (2001), refiriéndose al estudio de Bernard (1976), establecen cuatro pautas para abordar los contenidos. Contemplan el valor científico, la exactitud y claridad de conceptos, la actualización de los datos, la estructuración de los contenidos y su ordenación lógica, y por último, la cantidad y calidad de elementos facilitadores.

Encontramos opiniones a favor y en contra de su utilización, como consideramos previamente, argumentando los beneficios de la aplicación del mismo o sus desventajas. Pero no hay duda de que su uso generalizado es una práctica habitual en la mayoría de los Centros de Educación Primaria, ya sea físicamente en la gestión de las sesiones, o por medio de las actividades propuestas por el maestro como producto de la exploración de diversas editoriales, o en su caso, como fruto de la experiencia de cursos académicos anteriores. Puesto que antaño el docente pudo contar con la referencia del libro de texto escolar, seleccionando aquellas actividades que mejor se adecuasen a su realidad educativa específica para generar el tratamiento de cada contenido o unidad didáctica.

Gutiérrez y Cansino (2001) proponen la adecuación a cuatro pautas para establecer la elección de los libros de texto escolares, contemplando la pertinencia del lenguaje usado, la aplicación científica y didáctica, la gestión de la información y el uso de ilustraciones.

La adecuación del lenguaje empleado en los libros escolares es primordial, dada su finalidad educativa, puesto que en caso de no estar adaptados al nivel madurativo de los discentes, no se establecerán nexos comunicativos, ya que no llegarán a interiorizar el significado de lo presentado. Por ende, se debe atender a los tecnicismos, a la estructura semántica de las oraciones, o el vocabulario empleado. En definitiva, si el autor se ha puesto en el lugar del discente a la hora de expresar los contenidos.

La validez científica, pero didáctica, es fundamental, puesto que se debe perseguir la equidad entre la validez científica y su tratamiento didáctico, considerando tanto la gradación de dificultad al abordar los contenidos como la coherencia entre todas las dimensiones musicales tratadas. Hay cierta sintonía con lo que Ivanova (2012) jerarquiza como función constructiva, desde el punto de vista de facilitar el tratamiento didáctico de los libros de texto escolares.

El tratamiento de la información está muy relacionado con el aspecto anteriormente mencionado, puesto que se debe perseguir la coherencia global del libro de texto escolar. Se puede presentar como texto abierto, es decir, que sirva para enlazar con otras fuentes de conocimiento, o bien como texto cerrado, acotando los contenidos como un todo, sin posibilidad de apertura a otras fuentes de conocimiento. Este aspecto lo trata Ivanova (2012) como función informativo-cognitiva, aludiendo a la interpretación de los autores cuando desarrollan los contenidos del mediador didáctico.

Se debe abordar la información sin descuidar ninguna dimensión de la educación musical (arte y la cultura, audición musical, expresión instrumental y vocal, el lenguaje musical y el movimiento y la danza), con actividades enfocadas a potenciar la creatividad de los educandos, implicando un tratamiento interdisciplinar, contemplando una ejemplificación y utilidad para aplicar los nuevos conocimientos en la vida real, y teniendo siempre presente las ideas previas de los alumnos como punto de partida.

En relación alas ilustraciones de los textos, consideramos que son fundamentales, pero no sólo restringidas a las físicas del propio libro de texto, como partituras, musicogramas, etc., sino enlazando con las nuevas tecnologías, como por ejemplo, con el uso del libro digital por medio de la pizarra digital interactiva. Efectivamente, ya que pueden servir como elemento motivador en el proceso de enseñanza y aprendizaje, donde los discentes sean los protagonistas de su propio quehacer cognitivo, puesto que debemos tener en cuenta la 
convivencia diaria de los alumnos con las Tecnologías de la Información y la Comunicación - TICs(BOTELLA; GIMENO, 2014).

En la misma línea, Monreal et al. (2015) señalan la necesidad expresada por Marqués (2004) de que los docentes deben concienciarse para adaptar su praxis educativa a las posibilidades didácticas que posibilitan actualmente las TICs. Además, ellos estarían en consonancia con la función sugestiva, terminología utilizada por Ivanova (2012), considerando una necesidad imperativa que el libro de texto se muestre cautivador para los discentes, aprovechando las ilustraciones para tal finalidad.

Cabero et al. (1995) aludiendo al estudio de Richaudeau (1981) describen unas características a tener en cuenta en los libros de texto, atendiendo al rigor de los contenidos, a su adaptación a los distintos contextos particulares, a la claridad y diseño de los materiales, y a la coherencia pedagógica.

Por otra parte, Ivanova (2012) expresa la idea de función diagnóstica, donde se aborda la evaluación de los contenidos, como, por ejemplo, mediante la realización de actividades que inciten a la autoevaluación del discente, aspecto no tratado directamente por Gutiérrez y Cansino (2001).

Martínez (1992) considera los siguientes descriptores temáticos para la ficha de materiales: el modelo de escuela, el desarrollo del currículum, la organización escolar, la evaluación, la investigación e innovación, los códigos de organización del contenido, las relaciones con el entorno, los medios y recursos y, los niveles educativos y ámbitos de implementación. Además muestra el modelo de análisis de libros de texto que parte de nueve descriptores temáticos de la ficha de materiales, sentando las siguientes siete dimensiones de referencia para el análisis del material curricular (MARTÍNEZ, 1992, p. 15-18):

Qué modelo pedagógico sugiere el material. Finalidades educativas y principios curriculares.

Qué contenidos culturales se seleccionan y cómo se presentan. Código de selección y lógica de secuenciación y estructuración. Política de inclusiones y exclusiones de contenido. Cultura y valores.

Qué estrategias didácticas modela. Cuál es la instrumentalización metodológica de la transmisión cultural.

Cuál es el modelo de profesionalidad docente implícito en el material.

Cuál es el modelo de aprendizaje del estudiante.

Tareas organizativas que implican al centro.

Evaluación del material y su vinculación con programas de formación del profesorado.

Para concluir, Blanco (1994), establece de modo muy pormenorizado la división entre aspectos generales, estructura temática y lenguaje. Sin embargo, no abordan una concreción en un área de conocimiento o etapa educativa específica, a diferencia de los modelos de análisis tratados previamente, que sí se refieren especialmente a educación musical aunque también sirven de referencia para los docentes de otras áreas de conocimiento.

\section{Conclusiones}

En el presente trabajo se ha efectuado un análisis de las conexiones entre la educación y los libros de texto escolares, identificando sendos conceptos y sus características, para así comprender el tratamiento de dichos mediadores didácticos a lo largo de la historia del sistema educativo español, constatando la importancia otorgada y su concreción en la legislación educativa. Además, existen diferentes propuestas de análisis de libros de texto, 
fundamentalmente de educación musical, con la finalidad de servir de referencia para su elección.

A partir del binomio educación-libros escolares, determinamos las implicaciones educativas en los libros de texto de música, así como las inferencias generadas tras su empleo, ya que es el material curricular usado tradicionalmente en mayor medida en el proceso de enseñanza y aprendizaje. Por ello, se identifica la relevancia que suscita en los maestros, puesto que dependiendo de su aplicación puede llevar a condicionar la praxis educativa, por lo que acaecen consideraciones antagónicas sobre su valía, distinguiendo entre docentes conservadores y progresistas. Por ende, se concluye que el problema no radica exclusivamente en la calidad del libro de texto escolar, sino en un uso inapropiado, condicionado por una excesiva subordinación o una deficiente elección.

Tras la revisión de los preceptos educativos de la historia del sistema educativo español hasta la promulgación de la Ley Orgánica 1/1990, de 3 de octubre, de Ordenación General del Sistema Educativo, que establece la inclusión de la música oficialmente en el currículo de la etapa de Educación Primaria, en pleno asentamiento democrático español, hemos descubierto como inferencia directa, la primera alusión a los libros de texto escolares referida a la instrucción primaria, en el Reglamento Provisional de las Escuelas Públicas de Instrucción Primaria Elemental de 1838.

En el estudio de legislación educativa existen variadas referencias a los libros de texto, presentando una mayor cuantía aquellas que versan sobre su regulación. Sin embargo, no hemos encontrado alusión explícita a los libros escolares de índole musical.

Por otra parte, cabe destacar el interés mostrado por parte del Ministerio de Educación Nacional por su mejora, incentivando mediante concursos y premios de carácter anual a las mejores ediciones, como se expresa en la Ley de 17 de julio de 1945 sobre Educación Primaria. Además, se resalta el tratamiento compartido de materiales y libros de texto, puesto que propicia una aproximación al trabajo cooperativo, como apreciamos tanto en la Orden de 17 de enero de 1981, como en la Orden de 6 de mayo de 1982 y en la Orden de 25 de noviembre de 1982.

Por último, se ha presentado la dimensión del libro de texto de música como mediador didáctico, por lo que se han estudiado y comparado las pautas fijadas en los principales trabajos sobre análisis de libros de texto de música, así como en los instrumentos globales de análisis de libros de texto y material curricular. Por ende, se ha pretendido generar una visión plural, aportando una mayor riqueza y variedad, y de este modo, establecer un marco de referencia con los aspectos más relevantes, para facilitar a los docentes el proceso de selección de los libros de texto escolares de música, y así contribuir a la mejora educativa.

\section{Notas}

1 La educación obligatoria en España abarca dos etapas educativas, destinadas a todos los niños y niñas con edades comprendidas entre los seis y los dieciséis años.En primer lugar hallamos la Educación Primaria, dirigida a los educandos de seis a doce años. Posteriormente, se da continuidad con la etapa de Educación Secundaria Obligatoria, para discentes de entre doce y dieciséis.

2 Entendemos por currículo oculto, aquel que se aborda generalmente de modo inconsciente, por lo que se muestra excluido del currículo explícito y, atiende fundamentalmente a los valores, actitudes, normas, acciones y sentimientos. 3 ANELE, Asociación Nacional de Editores de Libros y Material de Enseñanza de España con sede en Madrid, aglutina al 96\% de los editores españoles de libros y materiales dirigidos a la enseñanza reglada no universitaria. Su finalidad radica en representar a los editores de libros y materiales educativos ante las entidades administrativas y, asesorar y coordinar sus actividades colectivas.

4 En todos los textos legislativos se ha respetado el formato de escritura original, sin incluir [sic], para preservar la esencia de los mismos.

5 Se denomina Plan Pidal al Plan General de Estudios para la instrucción pública promulgado por el Real Decreto 
de 17 de septiembre de 1845, porque dicho Real Decreto fue rubricado por el Ministro de la Gobernación de la Península, D. Pedro José Pidal. Con este Plan de Estudios se pretende una reorganización y centralización de los estudios bajo la directriz gubernamental.

6 La Dictadura franquista se establece durante el periodo comprendido entre los años 1939 y 1975. Estas fechas se enmarcan desde el final de la Guerra Civil Española, el 1 de abril de 1939, con el último parte de guerra rubricado por Francisco Franco donde se declara el fin de la misma y, por ende, la victoria del Bando Nacional, hasta su muerte, el 20 de noviembre de 1975.

7 Ley de 20 de septiembre de 1938 sobre Reforma de la Segunda Enseñanza, BOE 85, 1938, p. 1385-1395.

8 En la Base IV de la Ley de 20 de septiembre de 1938 sobre Reforma de la Segunda Enseñanza, se establecen como disciplinas de carácter fundamental las siguientes: Religión y Filosofía, Lenguas Clásicas, Lengua y Literatura Española, Geografía e Historia, Matemáticas, Lenguas Modernas, y Cosmología.

9 Ley de 17 de julio de 1945 sobre Educación Primaria, BOE 199, 1945, p. 385-416.

10 Ley 14/1970, de 4 de agosto, General de Educación y Financiamiento de la Reforma Educativa, BOE 187, 1970, p. $12525-12546$.

11 Real Decreto 69/1981, de 9 de enero, de ordenación de la Educación General Básica y fijación de las enseñanzas mínimas para el Ciclo Inicial, BOE 15, 1981, p. 1096-1098.

12 Real Decreto 710/1982, de 12 de febrero, por el que se fijan las enseñanzas mínimas para el ciclo medio de la Educación General Básica, BOE 90, 1982, p. 9586-9589.

13 Orden de 6 de mayo de 1982, por la que se regulan las enseñanzas del Ciclo Medio de la Educación General Básica, BOE 115, 1982, p. 12574-12588.

14 Real Decreto 3087/1982, de 12 de noviembre, por el que se fijan las enseñanzas mínimas para el ciclo superior de Educación General Básica, BOE 280, 1982, p. 32011-32017.

15 Ley Orgánica 1/1990, de 3 de octubre, de Ordenación General del Sistema Educativo, BOE 238, 1990, p. 2892728942.

\section{Referencias}

ADELL, Jordi. y BERNABÉ, Iolanda. Los libros de texto de la escuela en red. Perspectiva CEP, Sevilla, n. 11,p. 21-33, 2006.

ATIENZA, Encarna. Discurso e ideología en los libros de texto de ciencias sociales. Discurso \& Sociedad, Barcelona, v. 1, n. 4, p. 543-574, 2007.

BARNIOL i TERRICABRAS, Enriqueta. Pensamiento pedagógico y acción educativa de Zoltán Kodàly. Música y educación, Madrid, v. 11, n. 35, p. 85-102, 1998.

BERNABEU, José. L. Educación y dimensiones de la educación. En: COLOM, Antonio. J. (Coord.). Teorías e instituciones contemporáneas de la educación. Barcelona: Ariel, 1998. p. 17-34.

BLANCO, Nieves. Materiales curriculares: los libros de texto. En: ANGULO, José. F. y BLANCO, Nieves. (Coords.). Teoría y desarrollo del curriculum. Archidona, Málaga: Aljibe, 1994. p. 263279.

BOTELLA, Ana. M. y GIMENO, José V. Música de cine y audición en los libros de texto de enseñanza secundaria: consideraciones sobre su didáctica. Espiral. Cuadernos del Profesorado, Almería, v. 7, n. 14, p. 36-43, 2014.

BRAGA, Gloria. y BELVER, José. L. El análisis de libros de texto: una estrategia metodológica en la formación de los profesionales de la educación. Revista complutense de educación, Madrid, v. 27, n. 1, p. 199-218, 2016.

CABERO, Julio., DUARTE, Ana. y ROMERO, Rosalía. Los libros de texto y sus potencialidades para el aprendizaje. En: CABERO, Julio. y VILLAR, Luis. M. (Coords.). Aspectos críticos de una reforma educativa. Sevilla: Universidad de Sevilla, 1995. p. 21-39.

CABRERA, Isaac. El análisis de contenido en la investigación educativa: propuesta de fases y procedimientos para la etapa de evaluación de la información. Pedagogía Universitaria, La Habana, v. 14, n. 3, p.71-93, 2009. 
CARBONELL, Jaume. La aventura de innovar. El cambio en la escuela. Madrid: Morata, 2001.

DOMINGO, Ángel. El centro educativo: contexto social, político y normativo. La gestión de centros. En: ION, Georgeta. y DOMINGO, Ángel. (Eds.). Organización del Centro Escolar. Barcelona: FUOC, 2009. p. 1-51.

ESPAÑA, Jefatura del Estado, JE. Ley 14/1970, de 4 de agosto, General de Educación y Financiamiento de la Reforma Educativa. Boletín Oficial del Estado, BOE, 187 (6 de agosto), p. 12525-12546, 1970. Disponible en https://www.boe.es/boe/dias/1970/08/06/pdfs/A12525-12546. pdf Acceso el 22 nov 2018.

ESPAÑA, Jefatura del Estado, JE. Ley Orgánica 1/1990, de 3 de octubre, de Ordenación General del Sistema Educativo. Boletín Oficial del Estado, BOE, 238 (4 de octubre), p. 28927-28942, 1990. Disponible en https://www.boe.es/boe/dias/1990/10/04/pdfs/A28927-28942.pdf Acceso el 22 nov 2018.

ESPAÑA, Ministerio de Educación, ME. Orden de 17 de enero de 1981, por la que se regulan las enseñanzas de Educación Preescolar y del Ciclo Inicial de la Educación General Básica. Boletín Oficial del Estado, BOE, 18 (21 de enero), p. 1384-1389, 1981. Disponible en https://www.boe. es/boe/dias/1981/01/21/pdfs/A01384-01389.pdf Acceso el 22 nov 2018.

. Real Decreto 69/1981, de 9 de enero, de ordenación de la Educación General Básica y fijación de las enseñanzas mínimas para el Ciclo Inicial. Boletín Oficial del Estado, BOE, 15 (17 de enero), p. 1096-1098, 1981. Disponible en https:/www.boe.es/boe/dias/1981/01/17/pdfs/ A01096-01098.pdf Acceso el 22 nov 2018.

ESPAÑA, Ministerio de Educación y Ciencia, MEC. Orden de 6 de mayo de 1982, por la que se regulan las enseñanzas del Ciclo Medio de la Educación General Básica. Boletín Oficial del Estado, BOE, 115 (14 de mayo), p. 12574-12588, 1982. Disponible en https://www.boe.es/boe/ dias/1982/05/14/pdfs/A12574-12586.pdf Acceso el 18 nov 2018.

. Orden de 25 de noviembre de 1982, por la que se regulan las enseñanzas del Ciclo Superior de la Educación General Básica. Boletín Oficial del Estado, BOE, 291 (4 de diciembre), p. 33446-33466, 1982. Disponible en https:/www.boe.es/boe/dias/1982/12/04/pdfs/A3344633466.pdf Acceso el 22 nov 2018.

. Real Decreto 710/1982, de 12 de febrero, por el que se fijan las enseñanzas mínimas para el ciclo medio de la Educación General Básica. Boletín Oficial del Estado, BOE, 90 (15 de abril), p. 9586-9589, 1982. Disponible en https://www.boe.es/boe/dias/1982/04/15/pdfs/A09586-09589. pdf Acceso el 22 nov 2018.

. Real Decreto 3087/1982, de 12 de noviembre, por el que se fijan las enseñanzas mínimas para el ciclo superior de Educación General Básica. Boletín Oficial del Estado, BOE, 280 (22 de noviembre), p. 32011-32017, 1982. Disponible en http:/www.boe.es/boe/dias/1982/11/22/pdfs/ A32011-32017.pdf Acceso el 22 nov 2018.

ESPAÑA, Ministerio de Educación Nacional, MEN. Ley de 20 de septiembre de 1938, sobre Reforma de la Segunda Enseñanza. Boletín Oficial del Estado, BOE, 85 (23 de septiembre), p. 1385-1395, 1938. Disponible en https://www.boe.es/datos/pdfs/BOE/1938/085/A01385-01395. pdf Acceso el 22 nov 2018.

. Ley de 17 de julio de 1945, sobre Educación Primaria. Boletín Oficial del Estado, BOE, 199 (18 de julio), p. 385-416, 1945. Disponible en https://www.boe.es/datos/pdfs/BOE/1945/199/ A00385-00416.pdf. Acceso el 22 nov 2018.

ESPAÑA, Ministerio de Fomento, MF. Ley de 9 de septiembre de 1857, de Instrucción Pública (Ley Moyano). Gaceta de Madrid, GM, 1710 (10 de septiembre), p. 1-3, 1857. Disponible en https://www.boe.es/datos/pdfs/BOE/1857/1710/A00001-00003.pdf Acceso el 22 nov 2018. 
. Ley de 2 de junio de 1868, de Instrucción Primaria. Gaceta de Madrid, GM, 156 (4 de junio), p. 1-4, 1868. Disponible en http://www.boe.es/datos/pdfs/BOE//1868/156/A00001-00004. pdf Acceso el 22 nov 2018.

ESPAÑA, Ministerio de la Gobernación de la Península, Sección de Instrucción Pública, MGPSIP. Real Decreto de 17 de septiembre de 1845, por el que se aprueba el Plan General de Estudios para la instrucción pública del reino en la parte relativa á las enseñanzas secundaria y superior. Gaceta de Madrid, GM, 4029 (25 de septiembre), p. 1-5, 1845. Disponible en http://www.boe.es/ datos/pdfs/BOE//1845/4029/ Acceso el 18 nov 2018.

ESPAÑA, Ministerio de Instrucción Pública y Bellas Artes, MIPBA. Real Decreto de 26 de octubre de 1901. Gaceta de Madrid, GM, 303 (30 de octubre), p. 497-499, 1901. Disponible en http://www.boe.es/datos/pdfs/BOE//1901/303/A00497-00499.pdf Acceso el 18 nov 2018.

. Ley de 27 de agosto de 1932, disponiendo que el Consejo de Instrucción pública se transforme en Consejo Nacional de Cultura, con las atribuciones que se le asignan. Gaceta de Madrid, GM, 254 (10 de septiembre), p. 1818-1820, 1932. Disponible en http://www.boe.es/ datos/pdfs/BOE//1932/254/A01818-01820.pdf Acceso el 18 nov 2018.

FREGA, Ana. L. Música para maestros. 4. ed. Barcelona: Graó, 1996.

GARCÍA, Francisco. J. y ARREDONDO, Herminia. La imagen de la música tradicional andaluza en los materiales curriculares de la educación primaria. Sevilla: Fundación Pública Andaluza Centro de Estudios Andaluces, 2006.

GUTIÉRREZ, Rosario. y CANSINO, José. I. Música y libro de texto. Música y educación, Madrid, v. 14, n. 47,51-65, p. 2001.

IBÁÑEZ, Álex., VICENT, Naiara. y ASENSIO, Mikel. Aprendizaje informal, patrimonio y dispositivos móviles. Evaluación de una experiencia en educación secundaria. Didáctica de las Ciencias Experimentales y Sociales, Valencia, n. 26, p. 3-18, 2012.

IVANOVA, Anelia. Los libros para la enseñanza musical en España y Bulgaria. Música y educación, Madrid, v. 25, n. 92, p. 96-109, 2012.

LÓPEZ, Ana. Libros de texto y profesionalidad docente. Avances en supervisión educativa: Revista de la Asociación de Inspectores de Educación de España, Madrid, n. 6, p. 1-13, 2007.

MARTENOT, Maurice. Principios fundamentales de formación musical y su aplicación. Madrid: Rialp, 1993.

MARTÍNEZ, Jaume. ¿Cómo analizar los materiales? Cuadernos de Pedagogía, Las Rozas, n. 203, p. 14-18, 1992.

.Los libros de texto como práctica discursiva. Revista de la Asociación de Sociología de la Educación (RASE),Valencia, v. 1, n. 1, p. 62-73, 2008.

MONREAL, Inés M., GIRÁLDEZ, Andrea. y GUTIÉRREZ, Alfonso. Uso e integración curricular de la pizarra digital interactiva (PDI) en la clase de música en primaria: un estudio de caso en Segovia. Multidisciplinary Journal of Educational Research, Barcelona, v. 5, n. 1, p. 82-104, 2015.

ORIOL, Nicolás. Metodología cuantitativa y cualitativa en la investigación sobre la formación inicial del profesorado de educación musical para primaria. Aplicación a la formación instrumental. Revista Electrónica Complutense de Investigación en Educación Musical, Madrid, v. 1, n. 3, p.1-63, 2004. 
PÉREZ, Ángel El aprendizaje escolar: de la didáctica operatoria a la reconstrucción de la cultura en el aula. En: GIMENO, José. y PÉREZ, Ángel. (Coords.). Comprender y transformar la enseñanza. Madrid: Morata, 1993a. p. 63-77.

. Las funciones sociales de la escuela: de la reproducción a la reconstrucción crítica del conocimiento y la experiencia. En: Gimeno, José. y PÉREZ, Ángel. (Coords.). Comprender y transformar la enseñanza. Madrid: Morata, 1993b. p. 17-33.

ACERTAR FUENTE. Reglamento Provisional de las Escuelas Públicas de Instrucción Primaria Elemental de 1838, de 26 de noviembre. Madrid: Imprenta Nacional. Disponible en https://books.google.es/books?id=eo4fjeZ748EC\&pg=PA1\&hl=es\&source=gbs_selected_ pages\&cad $=2 \# \mathrm{v}=$ onepage \&q\&f$=$ false Acceso el 22 nov 2018.

SANUY, Montserrat. y GONZÁLEZ, Luciano. Orff-Schulwerk, música para niños (Versión original española basada en la obra de Carl Orff y GunildKeetman). Madrid: Unión Musical Española, 1969.

SARRAMONA, Jaume. La educación como proceso. En: COLOM, A.J. (Coord.). Teorías e instituciones contemporáneas de la educación. Barcelona: Ariel, 1998. p. 207-219.

SARRAMONA, Jaume. y RODRÍGUEZ, Teófilo. Participación y calidad de la educación. Aula Abierta, Oviedo, v. 38, n. 1, p. 3-14, 2010.

SCHIEFELBEIN, Ernesto. y FARRÉS, Pilar. Evaluación formativa de libros de texto de educación primaria. Revista Latinoamericana de Estudios Educativos, México, D. F., v. 21, n. 2, p. 63-87, 1991.

VICENTE, Gregorio. El libro de texto en educación musical. Espiral. Cuadernos del Profesorado, Almería, v. 3, n. 5, p. 30-35, 2010.

Sonsoles Ramos Ahijado. Doctora en Musicología por la Universidad de Salamanca. Licenciada en Historia y Ciencias de la Música. Diplomada en lengua extranjera: francés y diplomada en Educación Musical. Profesora Contratada Doctora del Departamento de Didáctica de la Expresión Musical, Plástica y Corporal en la Universidad de Salamanca, impartiendo docencia en la Escuela Universitaria de Educación (Campus de Ávila), y en la Facultad de Educación de Salamanca. En la actualidad es subdirectora de docencia práctica en la Escuela Universitaria de Educación y Turismo de Ávila, coordinadora del Grado en Maestro en Educación Primaria, coordinadora de la especialidad de Música del Máster Universitario Profesional de Educación Secundaria Obligatoria y Bachillerato. Sus líneas de investigación abarcan desde el uso del videojuego en el aula de música y la renovación de metodologías docentes hasta el folklore y las Tic`s.

Ana María Botella Nicolás. Profesora Contratada Doctora del Departamento de Didáctica de la Expresión Musical, Plástica y Corporal de la Facultad de Magisterio de la Universitat de València. Doctora en pedagogía por la Universitat de València. Es Licenciada en Geografía e Historia, especialidad Musicología y maestra en Educación Musical, por la Universidad de Oviedo. Grado profesional en la especialidad de piano y Máster internacional en la misma especialidad. Durante el año 2001 obtiene por oposición plaza en el cuerpo de profesores de música de enseñanza secundaria en Alicante. Forma parte de la Comisión de Coordinación Académica del Master Universitario en Profesor/a de enseñanza secundaria de la Universitat de València. Ha sido directora del Máster en Investigación en didácticas específicas. Coordina el doctorado en la especialidad de música de la Facultad de Magisteri. Ha sido directora del Aula de Música del Vicerrectorado de Cultura e Igualdad de la UVEG. Es Vicedecana de la Facultad de Magisterio. Sus líneas de investigación aúnan la didáctica de la historia de la música con las nuevas tecnologías, el folklore y la educación y las nuevas metodologías docentes.

José Luis Rodríguez Pérez. Doctor en Ciencias de la Educación (Departamento de Didáctica de la Expresión Musical, Plástica y Corporal. Universidad de Salamanca). Licenciado en Geografía e Historia en la especialidad de Musicología (Facultad de Geografía e Historia. Universidad de Salamanca). Diplomado en Profesorado de E.G.B. en la especialidad de Ciencias Humanas (Escuela Universitaria de Formación del Profesorado de E.G.B. de Teruel. Universidad de Zaragoza). Profesor Titular en el Centro de Magisterio Virgen de Europa, desde el curso académico 1995-96. La actividad investigadora: vinculada con la proyección educativa de la educación musical, principalmente focalizada en las etapas de Educación Primaria e Infantil. 